\title{
El desarrollo de competencias en los estudiantes de la facultad de contaduría-tuxpan a través de
}

\section{sus prácticas educativas.}

The development of competences in the students of the faculty of accounting-tuxpan through their educational practices.

Hidalgo Barrios Blanca Vianey ${ }^{1 凶}$, Gómez Priego Alejandra Ilean ${ }^{1}$, Elorza Martínez Oralia ${ }^{1}$, Camacho Díaz Othón Darío ${ }^{1}$ y Jonguitud Morales Norma Elisa ${ }^{1}$.

${ }^{1}$ Facultad de Contaduría. Campus Tuxpan. Universidad Veracruzana

${ }^{\circledR}$ Autor para correspondencia. E mail: vbarrios@hotmail.com

Recibido: $15 / 07 / 2017$

Aceptado: 10/12/2017

\section{RESUMEN}

Este artículo tiene como objetivo difundir la importancia de la enseñanza- aprendizaje realizado a través de las prácticas educativas. Este tipo de actividades permite al estudiante una interacción entre la teoría y la práctica en cada una de las experiencias educativas que cursa. Así como desarrollar distintas competencias de conocimiento, interpersonales y cognitivas que les ayudaran en su inserción laboral. Se ha beneficiado a 155 empresas de la Región de Poza Rica Tuxpan con este tipo de actividades docentes, como son la elaboración de Manuales, Diagnósticos, Planes, Programas y Proyectos, los cuales representan un instrumento de apoyo a todas estas empresas. La participación de los alumnos y maestros en este tipo de estrategias de aprendizaje ha sido muy enriquecedora, para los alumnos porque logran aprender y entender mejor los conocimientos teóricos y para los maestros el de convertirse en un guía que ayude a desarrollar sus competencias para que las apliquen en su vida personal y laboral.

Palabras clave: Prácticas Educativas, Estudiantes, Competencias, Empresas.

\begin{abstract}
ABSTRAC
This article aims to disseminate the importance of teaching and learning through educational practices. This type of activities allows the student an interaction between theory and practice in each of the educational experiences that he / she experiences. As well as developing different competences of knowledge, interpersonal and cognitive that will help them in their labor insertion. 155 companies from the Region of Poza Rica Tuxpan have benefited from this type of teaching activities, such as the preparation of Manuals, Diagnoses, Plans, Programs and Projects, which represent a tool to support all these companies. The participation of students and teachers in this type of learning strategies has been very enriching for the students because they are able to learn and understand better the theoretical knowledge and for the teachers to become a guide that helps to develop their competences so that the apply in your personal and work life.
\end{abstract}


Keywords: Educational Practices, Students, Competences, Companies.

\section{INTRODUCCIÓN}

Desarrollarse como docente va más allá de estar en un aula, es implementar las mejores estrategias para que los estudiantes capten y entiendan el conocimiento de sus experiencias educativas, al aplicar las prácticas académicas en sus experiencias educativas que imparten, no solo estamos atendiendo una exigencia sino que acercamos a nuestros estudiantes a un mundo laboral, que desea egresados altamente capacitados tanto en conocimiento como en habilidades, destrezas y actitudes, esta es una herramienta docente que permite adquirir las competencias que se necesitan en los profesionistas del futuro.

De acuerdo al enfoque por competencias, estas estrategias le proporcionan al alumno los elementos que le permitan enfrentar los retos de la vida con conocimientos teóricos y prácticos de manera eficiente, para poder integrarse al mercado laboral con un perfil innovador y dentro de un panorama de competitividad donde la calidad del producto tanto humano como material son claves fundamentales del éxito personal y grupal empezando por lo educativo. (Javier).

\section{MATERIALES Y MÉTODOS}

Los estudiantes de los programas educativos de Contaduría, Gestión y Dirección de Negocios y Sistemas Computacionales de la Facultad de Contaduría Tuxpan han desarrollad diversas prácticas realizadas como producto en algunas de sus experiencias educativas, como son: Recursos Humanos, Gestión del Talento Humano, Alineación Estratégica, Desarrollo de Habilidades Directivas, Administración de Recursos Humanos, Administración Contemporánea, Estrategias Comerciales, Recursos Humanos, Administración de las Tics y Proyectos de Comercio Exterior. En el desarrollo de estas prácticas educativas, ellos aplican sus conocimientos y desarrollan competencias.

Las competencias que se han logrado desarrollar en los estudiantes que realizan este tipo de prácticas son: Competencias académicas (en cuanto a su formación teórica y práctica), Competencias Interpersonales (como la expresión oral y escrita, liderazgo y trabajo en equipo) y las Competencias Cognitivas (que van desde la toma de decisiones, razonamiento, creatividad y pensamiento crítico).

Los estudiantes han realizado distintos proyectos derivados de estas prácticas, como son la elaboración de: Manuales de recursos humanos, Diagnostico de clima organizacional, Manuales de gestión del talento humano, Programas motivacionales y de Responsabilidad Social, Diagnósticos de ética y responsabilidad social, Programas de alineación estratégica, Planes de mercadotecnia, Planes de exportación y Proyectos de innovación administrativa. Cada uno de estos proyectos ha sido realizado en base a saberes teóricos incluidos en cada una de las experiencias educativas que cursan, indicándoles el procedimiento a seguir para su elaboración.

\section{RESULTADOS}

Los beneficios que se han obtenido mediante el desarrollo de este tipo de estrategia educativa que aplican los docentes en algunas de sus experiencias educativas son muchos, pero en primer lugar está el beneficio hacia los estudiantes, ya que les permite verificar los conocimientos adquiridos en la universidad para resolver problemáticas o necesidades en las empresas así como de incrementar la confianza en los empresarios por la educación proporcionada por las Universidad Veracruzana. Con este tipo de actividades se ha logrado beneficiar desde julio 2010 hasta julio 2017 a 155 empresas en Tuxpan, Poza Rica, Martínez de la Torre y Tihuatlan, Veracruz. 


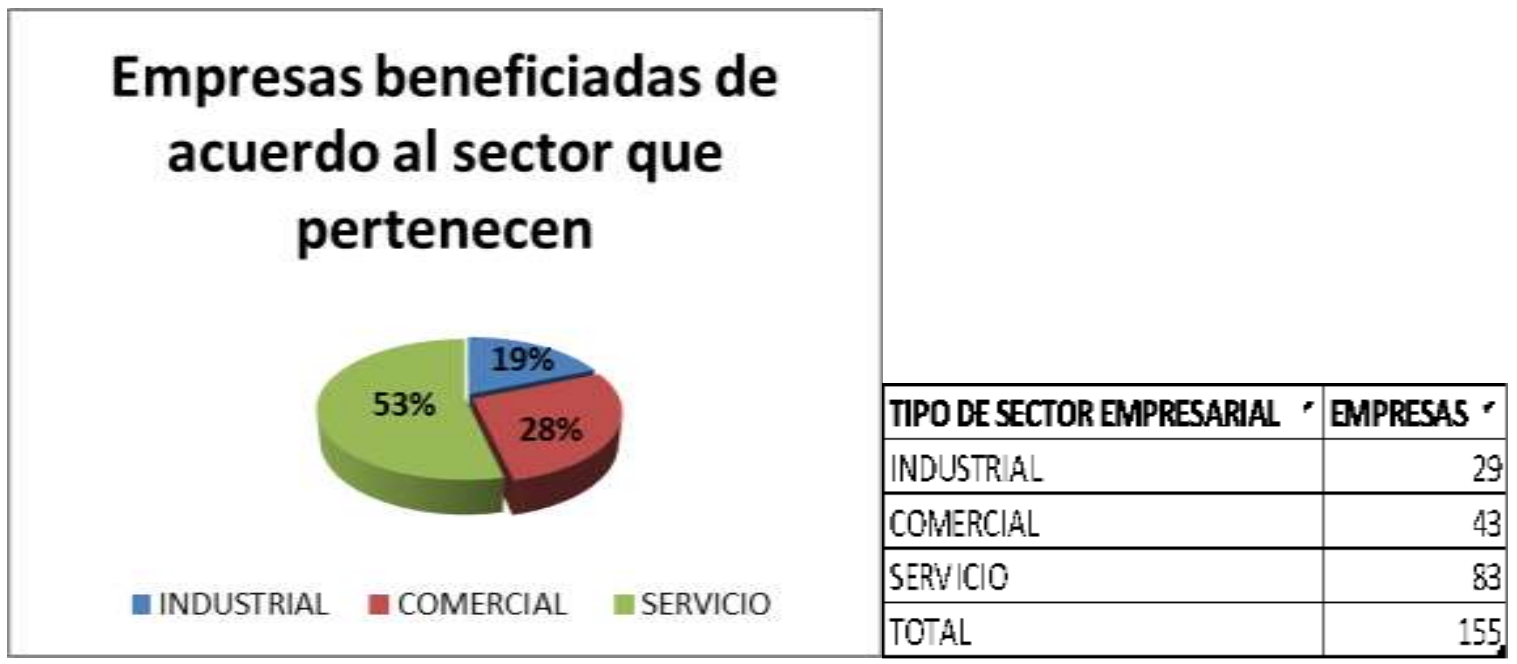

Grafico 1. Empresas de acuerdo al sector que pertenecen.

La Grafica 1 explica la participación de empresas beneficiadas en base al sector que pertenecen y en este caso la mayoría está representada por el $53 \%$ que corresponde a empresas del sector de servicio, un $19 \%$ empresas del sector industrial y el $28 \%$ son del sector comercial. Estas actividades prácticas realizadas con las empresas en distintas empresas de la región y que surgen a partir de sus clases en las experiencias educativas que cursan en la Facultad de Contaduría, ha generado la participación de estudiantes de los tres programas educativos: Contaduría, Gestión y Dirección de Negocios y Sistemas Computacionales Administrativos.

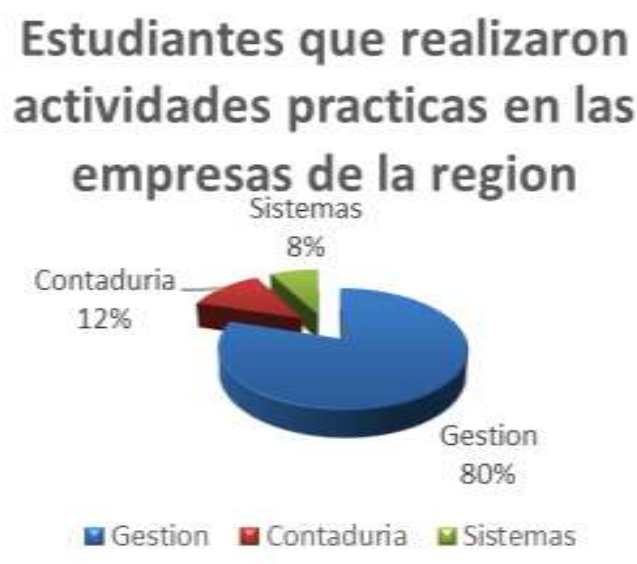

\begin{tabular}{|l|c|}
\hline PROGRAMAS EDUCATIVOS & ACTIVIDADES PRACTICAS \\
\hline Gestion & 124 \\
\hline Contaduria & 19 \\
\hline Sistemas & 12 \\
\hline Total & 155 \\
\hline
\end{tabular}

Grafica 2. Estudiantes por Programa Educativo 
La Grafica 2 nos muestra que la mayoría de estudiantes que han participado en estas activadas prácticas corresponde a los estudiantes de la Licenciatura en Gestión y Dirección de
Negocios, representando el $80 \%$, un $12 \%$ de la Licenciatura en Contaduría y solo un $8 \%$ estudiantes de la Licenciatura en Sistemas Computacionales Administrativos

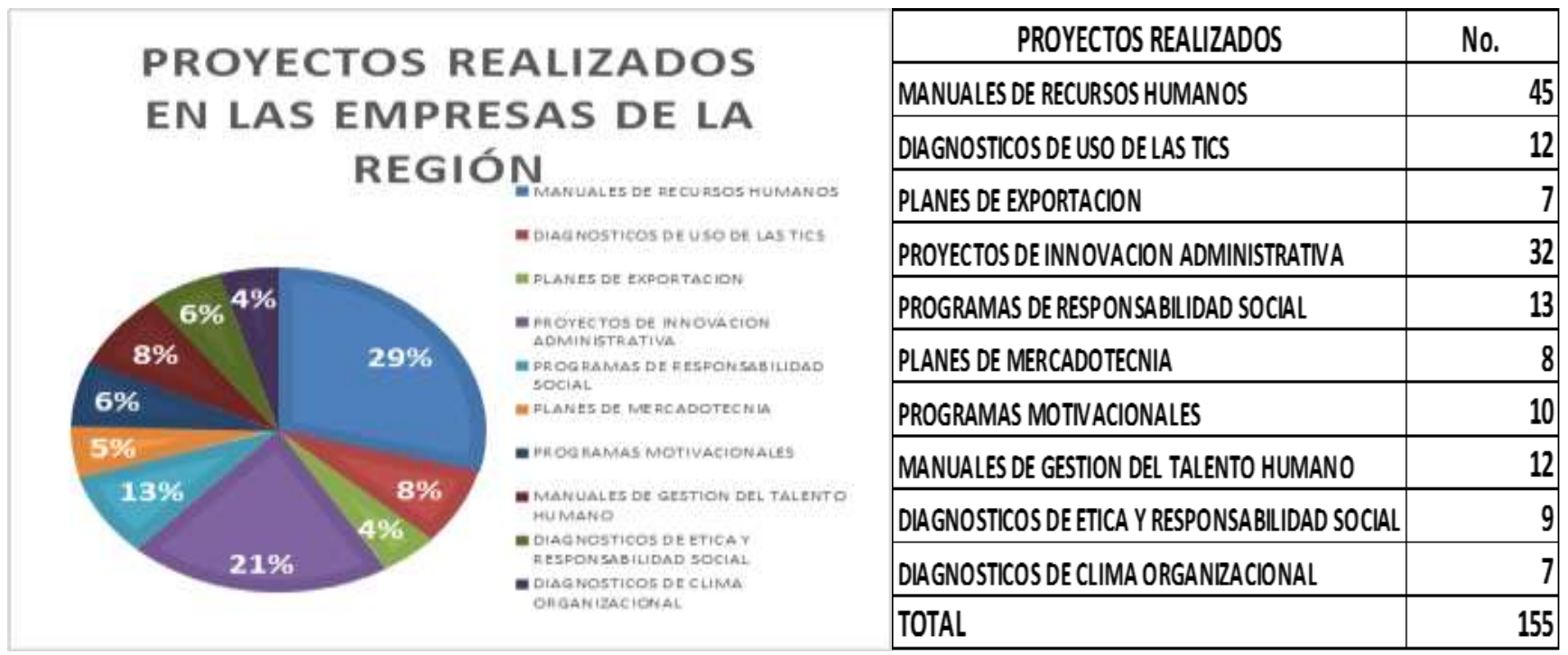

Grafica 3. Tipos de proyectos realizados en las empresas de la Región

Se han realizado hasta la fecha 155 proyectos a un mismo número de empresas, como se muestra en la gráfica 3, donde el mayor porcentaje está representado por la elaboración de Manuales de Recursos Humanos en un 45\%, Proyectos de innovación administrativa con el $32 \%$ y en una menor proporción los diagnósticos de clima organizacional y planes de exportación con el $7 \%$.

El desarrollo de estas prácticas educativas genera un impacto social muy importante, porque se refuerza la vinculación con los sectores productivos de la sociedad, además los empresarios van conociendo la capacidad de desempeño y habilidad de nuestros estudiantes, proporcionándoles herramientas que les permitan la inserción en su campo laboral.

\section{TESTIMONIO DE LOS ESTUDIANTES}

Para conocer la opinión de los estudiantes que realizaron estas prácticas educativas, se realizó una entrevista de manera aleatoria, se les pregunto que cuales fueron las competencias que desarrollaron al realizar este tipo de estrategia educativa, la mayoria de las respuestas se enfocan a que les ha permitido desarrollar habilidades, actitudes y reforzar valores para el compromiso, aprenden a trabajar en equipo, agilizan sus habilidades de 
comunicación, piensan críticamente, adquieren confianza en ellos y sobretodo entienden mejor lo aprendido en sus experiencias educativas.

Otra de las preguntas realizadas a los estudiantes fue que cual era su opinión acerca de los beneficios obtenidos por la elaboración de los proyectos educativos, obteniéndose como respuesta, que les permitió acercarse a la vida laboral, les ayudo a adquirir confianza y seguridad en sus conocimientos de la experiencia educativa, conocer el mundo empresarial, aplicar lo aprendido en el curso mediante la práctica y sobre todo brindar apoyo a las empresas para la solución de sus problemáticas o necesidades que se les presenten.

\section{CONCLUSIONES}

Las organizaciones de los sectores productivos del país, tienen una gran necesidad en sus empresas, derivado de muchas situaciones que se les presentan día con día, por lo que requieren de apoyo en algunas de sus actividades y es aquí donde los estudiantes pueden integrarse como una oportunidad para la solución de sus problemáticas.
Los futuros profesionistas adquieren con estas prácticas educativas las competencias que exige el mercado laboral, se les está capacitando para enfrentarse a su campo de trabajo. El desarrollar estas prácticas educativas es un reto para los docentes porque implica salirse de lo que tiene acostumbrado, de su zona de confort, pero los resultados obtenidos son muy satisfactorios tanto para los estudiantes como para las docentes, por lo que vale la pena realizar el esfuerzo al aplicar este tipo de estrategias educativas.

\section{LITERATURA CITADA}

(estudiante)

(educativo)

(Camacho D. O., 2012)

(Hidalgo, Licenciatura en Gestion y Direccion de Negocios, 2010-2016)

(Hidalgo, Licenciatura en Gestion y Direccion de Negocios, 2012-2016)

(Hidalgo, Licenciatura en Gestion y Direccion de Negocios, 2013)

(Hidalgo, Licenciatura en Gestion y Direccion de Negocios, 2013)

(Hidalgo, Licenciatura en Gestion y Direccion de Negocios, 2013)

(Hidalgo, Licenciatura en Gestion y Direccion de Negocios, 2013)

(Hidalgo, Licenciatura en Contaduria, 2015-2017) 
Copyright (c) 2017 Blanca Vianey Hidalgo Barrios, Alejandra Ilean G ómez Priego, Oralia Elorza Martinez,

Othón Dario Camacho Diaz y Norma Elisa Jonguitud Morales

\title{
(c) (1)
}

Este tex to está protegido por una licencia licencia Creative Commons 4.0

\begin{abstract}
Usted es libre para Compartir -copiar y redistribuir el material en cualquier medio o form ato- y Adaptar el documento -remezclar, transformar y crear a partir del material- para cualquier propósito, incluso para fi nes comerciales, siempre que cumpla la condición de:

Atribución: Usted debe dar crédito a la obra original de manera adecuada, proporcionar un enlace a la licencia, e in dicar si se han realizado cam bios. Puede hacerlo en cualquier form a razonable, pero no de form a tal que sugiera que tiene el apoyo del licenciante o 10 recibe por el uso que hace de la obra.
\end{abstract}

Resumenclicicencia - Textocompletocielalicencia 